\title{
Reclassification of Trichoderma Viride (TNAU), the Most Widely Used Commercial Biofungicide in India, as Trichoderma Asperelloides
}

\author{
Prasun K. Mukherjee*, Arup K. Mukherjee and Sandhya Kranthi \\ Crop Protection Division, Central Institute for Cotton Research, Nagpur 440010, India
}

\begin{abstract}
Trichoderma "viride" TNAU is the most widely used biofungicide in India with more than 250 registered commercial formulations being available. We have studied the phylogenetic position of this strain using a molecular marker and determined that this commercial strain is, in fact, a strain of Trichoderma asperelloides, and not T. viride. The implications of these findings on the registration and commercial distribution of biofungicides based on this strain has been discussed.
\end{abstract}

Keywords: Trichoderma viride, T. asperelloides, biofungicides, formulations.

\section{INTRODUCTION}

Trichoderma spp. are widely used as commercial biofungicides the world over [1]. Correct identification is important for successful and safe use of these fungi, comprising of more than 200 defined species. This is especially important since many beneficial and harmful traits are species-, and often strain-specific. For example, Trichoderma brevicompactum produces trichothecins (mycotoxins), and some species like $T$. longibrachiatum and $T$. citrinoviride are reported as human pathogens [2-4]. Unfortunately, Trichoderma taxonomy, relied earlier on morphology, has been confusing. This situation prevails till date for many isolates that are widely used, including many that are deposited in type culture collections [5]. A well known example is Trichoderma viride, the type species of this genus. Back in 1939, Bisby [6] merged all Trichoderma species into $T$. viride with perfect stage in Hypocrea rufa. In 1969, Rifai [7] proposed T. viride as one of the 9 species aggregates, and since then all Trichoderma strains having globose, subglobose, or ellipsoidal warted conidia were identified as $T$. viride. Lieckfeldt $e t$ al. [8], based on morphological, physiological and molecular data, proposed a new species T. asperellum within $T$. viride aggregate, and recently, Samuels et al. [9], based on multilocus genealogies, along with morphological and proteome data proposed a new species $T$. asperelloides that is distinctly different from $T$. asperellum. Therefore, the original $T$. viride isolates now comprise of three distinct species- $T$. viride, T. asperellum and T. asperelloides. The species could be differentiated based on sequences of tef1 (translation elongation factor 1-alpha) or rpb2 (RNA polymerase B, subunit 2) genes. In their studies, Samuels et al. [9] reclassified many widely used "T. viride" strains as T. asperelloides. Some of the extensively used strains that have been reclassi

*Address correspondence to this author at the Crop Protection Division, Central Institute for Cotton Research, Nagpur 440010, India;

Tel: +91-7103-275538; Fax: +91-7103-275529;

E-mail: prasunmukherjee1@gmail.com fied are T. harzianum T103 [10], T. asperellum T44 [11], T. viride ATCC 52439 and TR 31 [12]. T. viride strain, isolated and developed at the Tamil Nadu Agricultural University, Coimbatore, India [13] is the most popular biofungicides approved by the Central Insecticides Board (CIB), Govt. of India, and is extensively marketed in India in the form of more than 250 commercial formulation products [14].

\section{MATERIALS AND METHODS}

For checking if this commercial strain is indeed T. viride, we amplified and sequenced the large intron of tef 1 gene using the primer pair EF1-728F and EF1-986R (one of the most reliable phylogenetic markers for identification of Trichoderma spp.- http://www.isth.info/tools/blast/markers.php). The sequences were compared with those of authentic sequences of $T$. viride, $T$. asperellum and T. asperelloides from NCBI GenBank. The phylogenetic analysis was performed on www.phylogeny.fr.

\section{RESULTS AND DISCUSSION}

Trichoderma taxonomy has been very dynamic and has seen a great deal of changes after the switch from sole morphological keys to a combination of morphology and molecular phylogeny. Since the genus represents species ranging from saprophytes to plant symbionts and mycotoxinproducers to human pathogens, correct identification, especially of the commercial strains, are of utmost importance. Moreover, many traits are species specific and hence correct identification is necessary to exploit these fungi in a commercial setting. In the present study, we have analysed a strain of Trichoderma that is sold widely in India as T. viride and a DNA-sequencing (tef1 gene)- based phylogeny revealed that this isolate is not $T$. viride but $T$. asperelloides (Fig. 1). We also performed this analysis on a commercial formulation based on T. viride TNAU isolate (Phytoguard $1 \%$ WP, Central Biotech, Nagpur, India) and the sequence was identical with that of the original TNAU isolate, thus confirming the identification. The tef1 large (4th) intron se- 


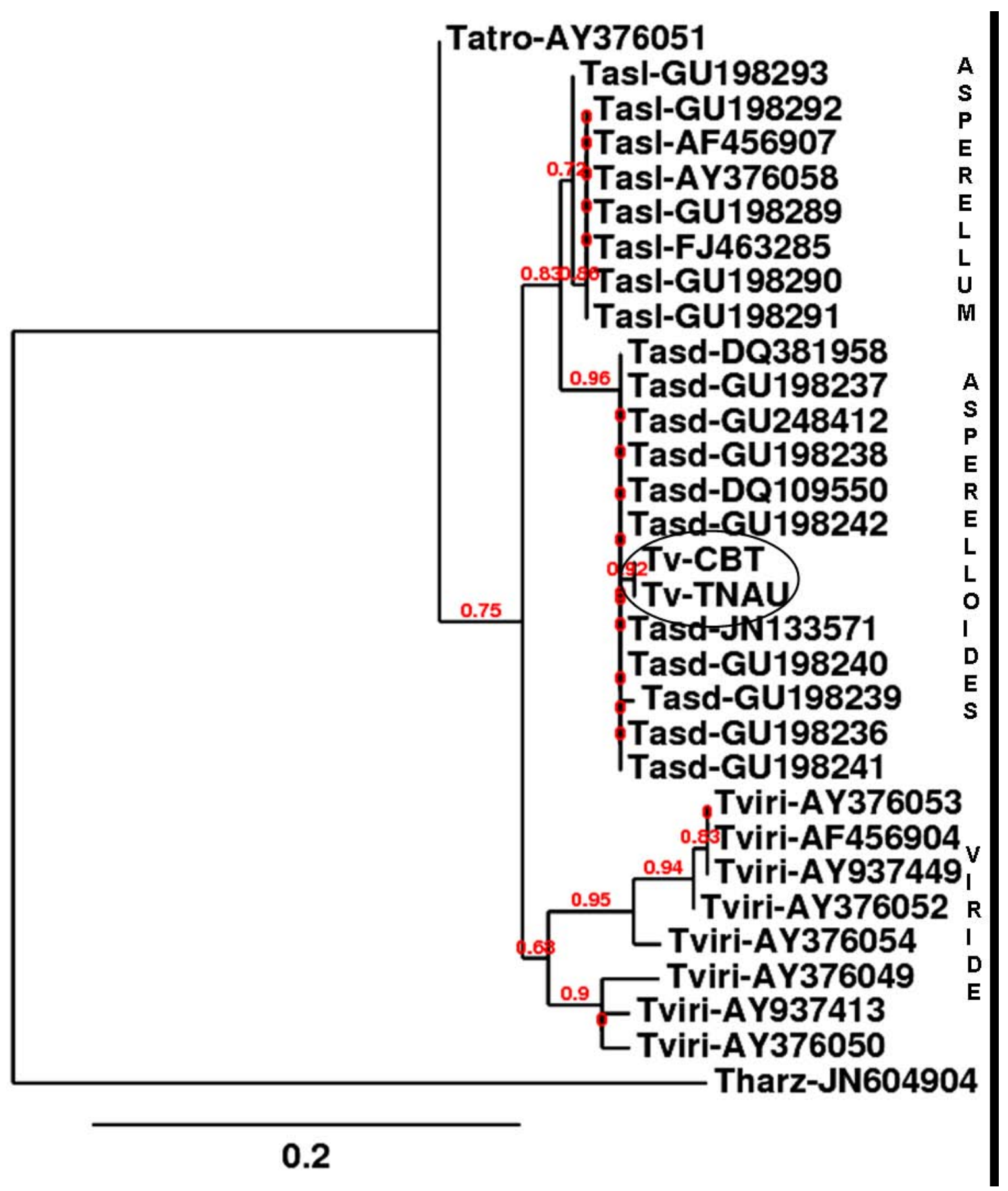

Fig. (1). Phylogenetic position of Trichoderma viride TNAU isolate (pure culture and commercial formulation, designated as TV-TNAU and Tv-CBT, respectively), relative to authentic T. viride, T. asperellum and T. asperelloides tef1 sequences. T. atroviride and T. harzianum sequences were used for comparison.

quence has been deposited with NCBI GenBank (accession no. KC679856). This finding necessitates the re-designation of more than 250 registered commercial formulations based on $T$. viride TNAU isolate as T. asperelloides.

\section{CONFLICT OF INTEREST}

The authors confirm that this article content has no conflicts of interest.

\section{ACKNOWLEDGEMENTS}

The authors gratefully acknowledge the financial support from the Govt of India in form of the Technology Mission in Cotton: Mini Mission-1(TMC MM-1) and Dr. K.R. Kranthi, Director, CICR, Nagpur for encouragement and support. 


\section{REFERENCES}

[1] Verma M, Brar SK, Tyagi RD, Surampalli RY, Valéro JR. Antagonistic fungi, Trichoderma spp.: Panoply of biological control. Biochem Eng J 2007; 37: 1-20.

[2] Nielsen KF, Gräfenhan T, Zafari D, Thrane U. Trichothecene production by Trichoderma brevicompactum. J Agric Food Chem 2005; 53: 8190-6.

[3] Kuhls K, Lieckfeldt E, Börner T, Guého E. Molecular reidentification of human pathogenic Trichoderma isolates as Trichoderma longibrachiatum and Trichoderma citrinoviride. Med Mycol 1999; 37: 25-33.

[4] Szekeres A, Láday M, Kredics L, et al. Rapid identification of clinical Trichoderma longibrachiatum isolates by cellulose-acetate electrophoresis-mediated isoenzyme analysis. Clin Microbiol Infect 2006; 12: 369-75.

[5] Latha J, Verma A, Mukherjee PK. PCR fingerprinting of some Trichoderma isolates from two Indian type culture collection-A need for reidentification of these economically important fungi. Curr Sci 2002; 83: 372-4.

[6] Bisby GR. Trichoderma viride Pers. Ex fries, and notes on $\mathrm{Hy}$ pocrea. Trans Br Mycol Soc 1939; 23: 149-68.

[7] Rifai MA. A revision of the genus Trichoderma. Mycol Pap 1969; 116: $1-54$.
[8] Lieckfeldt E, Samuels GJ, Nirenberg HI, Petrini O. A morphological and molecular perspective of Trichoderma viride: is it one or two species? Appl Environ Microbiol 1999; 65: 2418-28.

[9] Samuels GJ, Ismaiel A, Bon M-C, Respinis SD, Petrini O. Trichoderma asperellum sensu lato consists of two cryptic species. Mycologia 2010; 102: 944-66.

[10] Benhamou N, Chet I. Hyphal interactions between Trichoderma harzianum and Rhizoctonia solani: ultrastructure and gold cytochemistry of the mycoparasitic process. Phytopathology 1993; 83:1062-71.

[11] Sharon E, Chet I, Viterbo A, et al. Parasitism of Trichoderma on Meloidogyne javanica and role of the gelatinous matrix. Eur J Plant Pathol 2007; 118: 247-58.

[12] Papavizas GC, Lewis JA. Physiological and biocontrol characteristics of stable mutants of Trichoderma viride resistant to MBC fungicides. Phytopathology 1983; 73: 407-11.

[13] Sankar P, Jeyarajan R. Seed treatment formulation of Trichoderma and Gliocladium for biological control of Macrophomina phaseolina in sesamum. Indian Phytopathol 1996; 49:148-51.

[14] Singh HB, Singh BN, Singh SP, Sarma BK. 2012. Exploring different avenues of Trichoderma as a potent bio-fungicidal and plant growth promoting candidate-an overview. Rev Plant Pathol 2012; 5: 315-426.

Received: April 27, 2013

Revised: May 14, 2013

Accepted: May 16, 2013

(c) Mukherjee et al.; Licensee Bentham Open.

This is an open access article licensed under the terms of the Creative Commons Attribution Non-Commercial License (http://creativecommons.org/licenses/by-nc/3.0/) which permits unrestricted, non-commercial use, distribution and reproduction in any medium, provided the work is properly cited. 